\title{
Joint Routing, Scheduling, and Network Coding for Wireless Multihop Networks
}

\author{
Samat Shabdanov, Catherine Rosenberg, Patrick Mitran \\ Department of Electrical and Computer Engineering \\ University of Waterloo \\ Emails: sshabdan@engmail.uwaterloo.ca, cath@uwaterloo.ca,pmitran@ecemail.uwaterloo.ca
}

\begin{abstract}
This paper presents a study on achievable throughput in wireless multihop networks with unicast flows that use XOR-like network coding. A joint routing, scheduling, and network coding problem is formulated under a realistic signal to interference plus noise ratio interference model. This formulation provides a mathematical framework to study the achievable throughput of a given wireless network for a given utility function. We optimally solve it for $\max -\min$ throughput in small to medium size networks by developing an efficient computation tool. Our numerical results show that throughput gains can be obtained at low transmission powers by using simple XOR-like network coding in a mesh-like network provided it is optimally configured in terms of routing, scheduling, and network coding but that they are only significant (i.e., greater than 15\%) for some special cases. We also compute $\max -\min$ throughput by restricting network coding to some key nodes or flows to quantify key conditions that provide a significant portion of gains.
\end{abstract}

\section{INTRODUCTION}

In the last decades, interest in wireless multihop networks has grown rapidly due to their ability to increase network capacity and coverage, as well as to reduce energy consumption. Recently, industry has shown increased interest in multihop networks as they do not require additional infrastructure and have relatively low deployment costs. A multihop network typically consists of a gateway and multiple fixed mesh routers that aggregate the traffic from the end-users. The users and their mobility are not modeled in our framework, i.e., we consider the backhaul mesh network.

Multihop networks can be classified as a function of the type of their MAC protocol, i.e., random access or conflictfree scheduling [16]. In general, the throughput performance of conflict-free scheduling for high loads is much better than that of a random access MAC. In the following, we focus on centralized multihop networks using conflict-free scheduling [6]. Scheduling-based networks are important, since most of the new high data rates wireless technologies, such as WiMax and LTE, have an option for scheduling. Moreover, the achievable throughput for scheduling-based MAC can serve as an upper bound for any random access MAC [7]. Conflictfree scheduling MAC for multihop networks is based on spatial reuse TDMA access scheme (STDMA). The concept of scheduled STDMA was introduced in [15] as a channel access protocol. Since then, particular attention has been given to cross-layer optimization of routing and scheduling, as their joint optimization along with the configuration of some physical layer parameters such as power and rate shows significant network performance improvement [14].

Network Coding (NC) was introduced by [1] as a new network information theory technique. Since then, NC at- tracted a lot of attention primarily as an information theory field of study for networks [12], [20]. We study an XORlike network coding similar to COPE [9]. More precisely, we consider a simple linear NC without opportunistic listening, where intermediate nodes broadcast a combination of at most two packets. Packets are combined at intermediate nodes using a linear XOR operation and the original packets are decoded at each node that receives a coded packet (hop-byhop decoding). The initial motivation for this work is that while many experiments and testbeds have shown the potential benefits of network coding in term of throughput gains, no studies have shown how a network, jointly optimized for scheduling and routing, would compare to the same network jointly optimized for routing, scheduling, and network coding under a physical interference model. The main objective of this paper is to introduce a generic optimization framework for the joint routing, scheduling, and network coding problem for any given wireless multihop network with multiple unicast flows and to quantify gains that can be obtained by employing network coding. We want to emphasize that we have made the choice of a "simple" network coding in order to obtain an exact formulation that remains tractable (even if very complex) while not compromising on the interference model. It has been shown in [5] how simple interference models that yield conflict graphs can be qualitatively and quantitatively wrong. We believe that introducing optimal opportunistic listening to this exact formulation is too challenging and intractable.

In this paper, we present an optimization framework for the joint routing, scheduling, and network coding problem under a realistic signal to interference plus noise ratio interference (SINR) model (also called physical interference model) so as to quantify the gains that can be obtained by employing network coding. This problem is formulated for a general utility function and is solved for max - min throughput since as discussed in [14], it is a reasonable objective in the case of a managed mesh network. We focus on managed wireless multihop networks that are configured offline by their network operator to obtain the best throughput performance. We call network configuration the selection of the routing and scheduling parameters and possibly other parameters depending on the problem at hand. The main contributions of the paper are:

- We formulate a joint routing, scheduling, and XOR-like network coding problem based on the physical interference model in a network where all nodes use the same transmit power and the same modulation/coding scheme. The problem is NP hard and is much more complex than 
the problem introduced and solved in [14].

- We develop an efficient tool to compute exact solutions for small to medium-size networks in reasonable time when the utility function is the max - min throughput.

- Our formulation enables us to quantify the gains that can be obtained by using network coding for realisticsize mesh networks (i.e., networks containing a gateway whose flows are either destined to or from the gateway) as a function of the transmit power. We show that that they are only significant (i.e., greater than 15\%) at low to medium transmit powers for some special cases of midsized mesh-like networks (about 20 nodes), such as when the gateway is in a corner, which is typical of sectored systems.

- We also quantify the gains that can be obtained by restricting network coding to some nodes or by limiting network coding to only bidirectional flows. We find that similar gains can be achieved by these restricted variants of network coding. This has practical implications as optimizing a network with these restricted variants is significantly less complex than the original problem.

- A key conclusion of this study is that introducing the simplest form of network coding into a joint routing and scheduling framework under a realistic interference model yields a very high level of added complexity and hence there is little hope that such frameworks could be extended to more complex network coding scheme.

\section{RELATED WORK}

A theoretical framework for network coding was first developed by [1]. There, it was shown for a class of block codes, i.e., the so-called $\alpha$-codes, that it is theoretically possible to achieve multicast capacity by encoding messages with $\mathrm{NC}$ at intermediate nodes or relay nodes. This work was followed by [12], where network coding based on linear codes was proved to be sufficient to achieve maximum capacity for multicast traffic. In [9], the first system architecture using linear network coding was introduced. It was shown that employing network coding allows a significant increase of throughput for a wireless network with unicast traffic.

As an extension of the theoretical framework of [4], an asymptotic study of achievable throughput bounds in a wireless network using XOR-based network coding was presented by [13]. The bounds were derived for both protocol and physical interference models. In a network with network coding, the broadcast rate of linearly combined packets at a relay node is bounded by the lowest rate of the incoming links to the relay node. This limitation of network coding is addressed in the work of [20], where cooperative network coding is proposed to overcome the limitation imposed by the lowest rate link.

The work in [6] was the first optimization framework proposed to study the joint scheduling and routing problem for any given wireless network. Due to the complexity of the problem, the interference model used for computing all results presented therein was the protocol interference model. Furthermore, these results were limited to only lower and upper bounds on the throughput, and were only computed for small networks without considering multiple powers and rates for finding an optimal network configuration.

In the subsequent literature, simplified interference models such as the protocol interference model were widely used to study joint resource allocation problems. However, the validity of results based on these simplified interference models is questionable as discussed in [5] and [3]. It is widely accepted now that an additive SINR-based physical interference model should be used to model interference and to provide realistic insightful results.

The work of [6] was later extended by [7] to include power control and rate adaptation in the joint routing and scheduling optimization problem. In [7], a unified and general framework based on the physical interference model was presented and exact solutions for the case of a $\max -\min$ throughput for small to medium-size networks found.

Much research has been dedicated to developing efficient computational techniques for the cross-layer resource allocation problem. The work of [19] developed a computational technique based on a column generation method to solve a problem similar to the one in [6]. Later, the work of [19] was extended by [14], where an efficient enumeration algorithm and a column generation technique were proposed to solve the joint routing and scheduling problem for a network with power control and rate adaptation. This technique enabled the authors to provide exact solutions for large wireless networks for both $\max -\min$ and proportional fair throughput.

An information theoretical study of jointly optimized network coding and scheduling was presented in [16]. In [11], a cross-layer design of wireless multi-channel mesh networks is studied for a network code construction in a joint routing and MAC scheduling problem. The problem of rate control with pairwise inter-session network coding for distributed networks was formulated in [10] and in directed networks in [18].

So far, there has been little research in the area of joint optimization of network coding with routing and scheduling for wireless multihop networks. To the best of the authors knowledge, the work of [17] was the first significant contribution in this area. It tries to analyze joint routing and network coding for wireless unicast traffic. The proposed approach is an extension of the theoretical framework of [6] to allow for broadcast transmission and to optimize routing with $X O R$ based network coding with and without opportunistic listening, initially presented in [8]. The study in [17] has a number of limitations: it is based on the protocol interference model, uses an approximate model for computing a broadcast rate and it only computes bounds. Moreover, the joint routing and network coding problem is formulated for a given set of predefined routing paths for each source node. In contrast, in this paper the link scheduling is jointly optimized with routing and network coding, is based on the physical interference model, and provide exact solutions.

\section{MODEL DESCRIPTION AND BACKGROUND}

A wireless mesh network is modeled with a set of nodes $\mathcal{N}$, a set of directed links $\mathcal{L}$ and a set of flows $\mathcal{F}$. Each flow $f \in \mathcal{F}$ is defined as $f=\left(f_{s}, f_{d}\right)$, where $f_{s}=o(f)$ is the source node 
and $f_{d}=d(f)$ is the destination node. We further assume that the traffic is static or quasi-static to enable a flow based model. All nodes transmit with the same power $P$ and use the same coding and modulation yielding without loss of generality a normalized transmission rate of $c=1$ on all feasible links. Each directed link $\ell \in \mathcal{L}$ is specified by a pair of nodes as $\ell=\left(\ell_{s}, \ell_{d}\right)$, where $\ell_{s}=o(\ell)$ is the origin and $\ell_{d}=d(\ell)$ is the destination node of link $\ell$, respectively. A link $\ell \in \mathcal{L}$ must satisfy $P G_{o(\ell), d(\ell)} / N_{0} \geq \beta$ to be feasible, where $N_{0}$ is the additive white Gaussian noise power, $G_{o(\ell), d(\ell)}$ is the channel gain over the link $\ell=(o(\ell), d(\ell))$, and $\beta$ is the minimum Signal-to-Noise Ratio (SNR) requirement for the rate $c$.

\section{A. Conflict-free Scheduling}

To describe the conflict-free scheduling mechanism under the physical interference model, the notion of independent set (ISet) needs to be introduced. An ISet $s$ is a set of links that can be scheduled at the same time such that each link $\ell$ in $s$ can transmit error-free with the link rate $c$. If each node is equipped with an omni-directional antenna and links are half duplex, then a set of links $s$ is an ISet iff each link $\ell \in s$ satisfies the necessary conditions (1) and (2).

$$
\begin{array}{cc}
o\left(\ell_{1}\right) \neq o\left(\ell_{2}\right) ; o\left(\ell_{1}\right) \neq d\left(\ell_{2}\right) ; & \forall \ell_{1}, \ell_{2} \in s \\
d\left(\ell_{1}\right) \neq d\left(\ell_{2}\right) & \ell_{1} \neq \ell_{2} \\
\gamma_{\ell}=\frac{P G_{o(\ell), d(\ell)}}{N_{0}+P \sum_{\substack{k \in s \\
k \neq \ell}} G_{o(k), d(\ell)}} \geq \beta & \forall \ell \in s
\end{array}
$$

Condition (1) specifies the requirement that no two distinct links can share a node (destination or source). Condition (2) states that the SINR $\gamma_{\ell}$ of each link $\ell \in s$ must be greater than $\beta$ so that each link in $s$ yields an error-free transmission with the rate $c$. In (2), $G_{o(k), d(\ell)}$ is the channel gain between nodes $o(k)$ and $d(\ell)$.

Now, having defined the notion of ISets under the physical interference model, we can define the link scheduling mechanism. Usually, in scheduled-based networks, a scheduling round or frame consists of a sequence of slots during which ISets are assigned for transmission. In such a time-slotted network the optimization of network capacity for optimal scheduling and routing parameters involves a binary problem formulation similar to [2]. To overcome the complexity of an integer problem formulation and provide bounds on achievable throughput in a realistic sized network, the length of the scheduling frame is assumed to be infinitely long to allow a fractional model similar to [6] and [14]. Therefore, the link scheduling is addressed by computing the fraction of time a particular ISet is scheduled yielding a non-integer flow-based program. More precisely, let us define $\alpha_{s}$ to be the fraction of time ISet $s \in \mathcal{I}$ is scheduled. A link schedule is a vector $\left(\alpha_{s}\right)_{s \in \mathcal{I}}$ such that $\alpha_{s}>0$ if ISet $s$ is scheduled, otherwise $\alpha_{s}=0$. By scheduling only ISets the schedule is conflictfree. The set of all possible ISets is denoted by $\mathcal{I}$ and $\mathcal{I}_{\ell} \subseteq \mathcal{I}$ is defined to be the set of ISets that contain a link $\ell$.

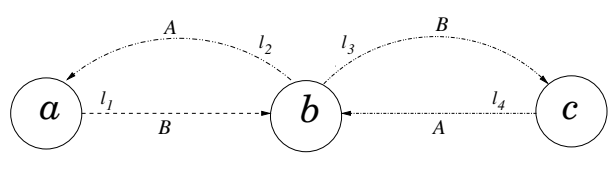

Fig. 1. Network without NC

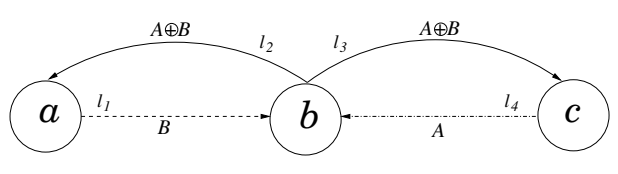

Fig. 2. Network with NC

\section{B. Network Coding Model}

We study an XOR-like network coding similar to COPE [9]. More precisely, we consider a simple linear $\mathrm{NC}$ without opportunistic listening, where intermediate nodes broadcast a combination of at most two packets. Packets are combined at intermediate nodes using a linear XOR operation and the original packets are decoded at each node that receives a coded packet (hop-by-hop decoding).

To illustrate the benefits of this NC technique as well as its impact on the notion of ISets, we consider a simple wireless multihop network with three nodes $\{a, b, c\}$ and a set of directed links $\mathcal{L}=\left\{\ell_{1}, . ., \ell_{4}\right\}$ as shown in Fig. 1. Assume that node $a$ wants to transmit a packet $B$ to $c$ and that node $c$ has a packet $A$ for node $a$. Node $b$ acts as a relay node between nodes $a$ and $c$. Let us assume also that the rate of each link is $c=1$ and no spatial reuse is possible. Hence, if network coding is not allowed, the only possible ISets are made of single links, i.e., $\mathcal{I}=\left\{\left\{\ell_{1}\right\}, \ldots,\left\{\ell_{4}\right\}\right\}$. Therefore, to send a packet $B$ from $a$ to $c$ and a packet $A$ from $c$ to $a$, four time units are required and a flow throughput of $1 / 4$ can be achieved (assuming 2 flows: $f_{1}=\{a, c\}$ and $f_{2}=\{c, a\}$ ).

Now, let us employ the simple $\mathrm{NC}$ technique from [9] without opportunistic listening. By using this technique, node $b$ can transmit a combination of two packets to its neighbors. Then, as shown in Fig. 2, instead of forwarding the individual packets, node $b$ transmits a packet $A \oplus B$. At nodes $a$ and $c$ this combination is decoded by using an $X O R$ operation with packet $B$ and $A$, respectively. This can be seen as allowing a new ISet $s=\left\{\ell_{2}, \ell_{3}\right\}$ (this will require a change in constraint (1) as will be seen later). Hence, this simple NC technique enables an increase of the potential spatial reuse of the system by producing additional ISets. The new set of ISets is now $\mathcal{I}=\left\{\left\{\ell_{1}\right\}, \ldots,\left\{\ell_{4}\right\},\left\{\ell_{2}, \ell_{3}\right\}\right\}$. Note that, as will be seen later, introducing $\mathrm{NC}$ in the joint optimization problem requires more than just increasing the set of ISets.

By enabling this $\mathrm{NC}$ technique only three time units are now necessary and this simple XOR-based network coding yields a throughput increase of $33 \%$ in this network.

\section{Joint Routing and Scheduling}

In this section, we describe the baseline formulation for the Joint Routing and Scheduling (JRS) problem without network coding. This formulation is extended later in Section IV to include the XOR-like network coding discussed above. 
Given $\mathcal{F}, \mathcal{L}, \mathcal{N}$, and $\mathcal{I}$ defined earlier, denote by $x_{f}(\ell)$ the amount of flow $f$ transmitted over a link $\ell$ (clearly a function of routing). Define $\lambda_{f}$ to be the rate of flow $f \in \mathcal{F}$. In order to simplify notation, we introduce the vectors $\boldsymbol{x}=\left\{x_{f}(\ell)\right\}_{\ell, f}$ and $\boldsymbol{\alpha}=\left\{\alpha_{s}\right\}_{s \in \mathcal{I}}$. Here, $\boldsymbol{\alpha}$ is a link scheduling vector and vector $\boldsymbol{x}$ represents an aggregated traffic/routing vector.

Given a utility function $\mathbf{U}\left\{\left(\lambda_{f}\right)_{f \in \mathcal{F}}\right\}$, the JRS flow-based problem can be formulated as follows[14]:

$$
\begin{gathered}
\max _{\boldsymbol{\alpha}, \boldsymbol{x}, \lambda} U\left(\left\{\lambda_{f}\right\}_{f \in \mathcal{F}}\right) \\
\sum_{\substack{\ell \in \mathcal{L} \\
o(\ell)=n}} x_{f}(\ell)-\sum_{\substack{\ell \in \mathcal{L} \\
d(\ell)=n}} x_{f}(\ell)=\left\{\begin{array}{ccc}
\lambda_{f}, & n=f_{s} \\
-\lambda_{f}, & n=f_{d} \\
0, & \text { else } & \forall \in \in \mathcal{F} \\
f \in \mathcal{F}
\end{array}\right. \\
\sum_{f \in \mathcal{F}} x_{f}(\ell) \leq \sum_{s \in \mathcal{I}_{\ell}} \alpha_{s} \\
\sum_{s \in \mathcal{I}} \alpha_{s}=1 \\
\boldsymbol{\alpha}, \boldsymbol{x} \geq 0
\end{gathered}
$$

Conditions (4) are the flow conservation constraints. Link scheduling constraints are given in (5) and (6). Specifically, (5) defines the link capacity constraints, i.e., that the aggregated amount of all flows over the link cannot exceed its scheduled capacity. The constraints (6) state that the ISets must be scheduled over a period of unit of time.

The problem (3)-(7) is a very large standard LP formulation whenever $U\left\{\left(\lambda_{f}\right)_{f \in \mathcal{F}}\right\}$ is linear. It was shown in [6] that it is NP-hard to find or even to approximate the optimal solutions for this type of LPs. In addition, the number of variables in the JRS problem grows exponentially with the network size. The work of [14] proposes efficient algorithmic techniques to compute the optimal (exact) solutions for relatively large networks (up to 60 nodes). In general, the optimal network configuration is not unique. By solving this problem, we not only obtain the optimal value of the objective function but also the optimal configuration of the network, i.e., the scheduling vector $\boldsymbol{\alpha}$ and the traffic/routing vector $\boldsymbol{x}$.

\section{Joint Routing, Scheduling, ANd Network CODING}

In this section, we formulate the joint routing, scheduling, and network coding (JRS-NC) problem based on the baseline formulation described in Section III-C. We aim to incorporate XOR-like network coding into the flow-based model by introducing new variables and NC constraints. The JRS-NC problem is formulated as a generic framework for a given wireless network and for a given utility function.

\section{A. JRS-NC Model and Definitions}

Recall that the network coding technique that we consider is without opportunistic listening and only allows coding between the data of two flows transiting through an intermediate relay node in opposite directions. For any $f_{1}, f_{2} \in \mathcal{F}$ and any $\ell_{1}, \ell_{2} \in \mathcal{L}$, denote by $y_{f_{1}, f_{2}}\left(\ell_{1}, \ell_{2}\right)$ the amount of flow $f_{1}$ transmitted over link $\ell_{1}$ and the amount of flow $f_{2}$ transmitted over link $\ell_{2}$ using NC. This new variable can only be defined if links $\ell_{1}$ and $\ell_{2}$ are such that $o\left(\ell_{1}\right)=o\left(\ell_{2}\right)$ and $\ell_{1} \neq \ell_{2}$. The variable $y_{f_{1}, f_{2}}\left(\ell_{1}, \ell_{2}\right)$ represents the amount of flow $f_{1}$ (resp. $f_{2}$ ) that is network coded and transmitted over link $\ell_{1}$ (resp. link $\ell_{2}$ ). To better illustrate it, consider Fig. 2. In this example only the following pair of $\mathrm{NC}$ variables can be defined: $y_{f_{2}, f_{1}}\left(\ell_{2}, \ell_{3}\right)=y_{f_{1}, f_{2}}\left(\ell_{3}, \ell_{2}\right)$ and $y_{f_{1}, f_{2}}\left(\ell_{2}, \ell_{3}\right)=y_{f_{2}, f_{1}}\left(\ell_{3}, \ell_{2}\right)$. Let $x_{f}(\ell)$ denote the amount of flow $f$ transmitted over link $\ell$ and not encoded with the $\mathrm{NC}$ technique.

The feasibility of ISets in the general JRS problem is constrained by the half-duplex condition given in (1). By allowing NC, an ISet $s$ can now contain two links that share a source node. Moreover, an ISet $s$ can contain multiple nodes that act as intermediate NC nodes. Hence, the definition of ISets $s$ when NC is enabled must be re-defined. A set of links $s$, in a network in which NC in enabled and defined as above, iff each link $\ell \in s$ meets the SINR condition given in (2) and the conditions in (8) and (9):

$$
\begin{array}{ll}
o\left(\ell_{1}\right) \neq d\left(\ell_{2}\right) ; d\left(\ell_{1}\right) \neq d\left(\ell_{2}\right) & \forall \ell_{1}, \ell_{2} \in s ; \ell_{1} \neq \ell_{2} \\
\sum_{\ell_{1} \in s} 1_{\left\{o\left(\ell_{1}\right)=o(\ell)\right\}} \leq 2 & \forall \ell \in s
\end{array}
$$

We define the network coding traffic/routing vector $\boldsymbol{y}=$ $\left[y_{f_{1}, f_{2}}\left(\ell_{1}, \ell_{2}\right)\right]_{f_{1}, f_{2}, \ell_{1}, \ell_{2}}$. Also, to simplify notation, let $\bar{\ell}$ be defined as the inverse link of link $\ell$, i.e., the link such that $o(\bar{\ell})=d(\ell)$ and $d(\bar{\ell})=o(\ell)$.

Let $\mathcal{I}_{\ell}$ be the set of ISets $s \in \mathcal{I}_{\ell}$ that contain link $\ell$ such that the node of origin $o(\ell)$ is not shared with any other link in ISet $\ell \in s$. Also define by $\mathcal{I}_{\ell_{1}, \ell_{2}}$ the set of all ISets $s \in \mathcal{I}_{\ell_{1}, \ell_{2}}$ that contain links $\ell_{1}$ and $\ell_{2}$ such that $o\left(\ell_{1}\right)=o\left(\ell_{2}\right)$ and $\ell_{1}, \ell_{2} \in s$, i.e., the set $\mathcal{I}_{\ell_{1}, \ell_{2}}$ defines all possible ISets that for which there is $\mathrm{NC}$ over the pair of links $\left(\ell_{1}, \ell_{2}\right)$.

\section{B. JRS-NC Formulation}

In our system, we allow all paths a priori and we allow each intermediate node to relay part of each flow it receives with $\mathrm{NC}$ and its remaining part without. Our optimization framework will compute the optimal amount of each flow to be carried on each feasible link and determine where and if $\mathrm{NC}$ is required.

The joint routing, scheduling and network coding problem for a network with the given sets $\mathcal{F}, \mathcal{L}, \mathcal{N}, \mathcal{I}_{\ell}, \mathcal{I}_{\ell_{1}, \ell_{2}}$ and for the utility function $U\left(\left\{\lambda_{f}\right\}_{f \in \mathcal{F}}\right)$ can be formulated as follows:

$$
\begin{aligned}
& \max _{\boldsymbol{\alpha}, \boldsymbol{x}, \boldsymbol{y}} U\left(\left\{\lambda_{f}\right\}_{f \in \mathcal{F}}\right) \\
& \sum_{\substack{\ell_{1} \in \mathcal{L} \\
o\left(\ell_{1}\right)=n}} x_{f_{1}}\left(\ell_{1}\right)-\sum_{\substack{f_{2} \in \mathcal{F} ; \ell_{1}, \ell_{2} \in \mathcal{L} \\
d\left(\ell_{1}\right)=n ; o\left(\ell_{2}\right)=o\left(\ell_{1}\right)}} y_{f_{1}, f_{2}}\left(\ell_{1}, \ell_{2}\right)- \\
& -\sum_{\substack{\ell_{1} \in \mathcal{L} \\
d\left(\ell_{1}\right)=n}} x_{f_{1}}\left(\ell_{1}\right)+\sum_{\substack{f_{2} \in \mathcal{F} ; \ell_{1}, \ell_{2} \in \mathcal{L} \\
o\left(\ell_{1}\right)=n ; o\left(\ell_{2}\right)=n}} y_{f_{1}, f_{2}}\left(\ell_{1}, \ell_{2}\right) \\
& =\left\{\begin{array}{cll}
\lambda_{f_{1}}, & n=o\left(f_{1}\right) \\
-\lambda_{f_{1}}, & n=d\left(f_{1}\right) \\
0, & \text { else } & \forall n \in \mathcal{N}, \\
0 & f_{1} \in \mathcal{F}
\end{array}\right.
\end{aligned}
$$




$$
\begin{aligned}
& \sum_{f \in \mathcal{F}} x_{f}(\ell) \leq \sum_{s \in \mathcal{I}_{\ell}} \alpha_{s} \quad \ell \in \mathcal{L} \\
& \sum_{f_{1}, f_{2} \in \mathcal{F}} y_{f_{1}, f_{2}}\left(\ell_{1}, \ell_{2}\right) \leq \sum_{s \in \mathcal{I}_{\ell_{1}, \ell_{2}}} \alpha_{s} \quad \forall \ell_{1}, \ell_{2} \in \mathcal{L} \\
& \sum_{f_{1}, f_{2} \in \mathcal{F}} y_{f_{1}, f_{2}}\left(\ell_{1}, \ell_{2}\right) \leq \sum_{f \in \mathcal{F}} x_{f}\left(\overline{\ell_{1}}\right)+ \\
& o\left(\ell_{2}\right)=o\left(\ell_{1}\right) \\
& +\sum_{f_{1}, f_{2} \in \mathcal{F} ; \ell_{2} \in \mathcal{L}} y_{f_{1}, f_{2}}\left(\bar{\ell}_{1}, \ell_{2}\right) \quad \ell_{1} \in \mathcal{L} \\
& o\left(\ell_{2}\right)=o\left(\overline{\ell_{1}}\right)
\end{aligned}
$$

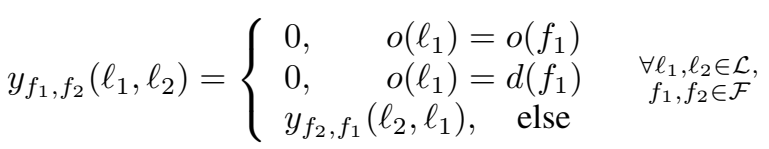

$$
\begin{aligned}
& \sum_{s \in \mathcal{I}} \alpha_{s}=1 \\
& \boldsymbol{\alpha}, \boldsymbol{x}, \boldsymbol{y} \geq 0
\end{aligned}
$$

Condition (11) specifies the flow conservation constraints, which requires the amount of flows with and without network coding to be conserved at any node $n$. Constraints (12) and (13) specify the link capacity requirements for the flows without and with network coding, respectively. For the description of link scheduling and other non-network coding constraints, we refer to the baseline framework discussed in Section III-C.

The NC constraints in (14) require that the total amount of network coded traffic transmitted over the link $\ell_{1}$ by a node $o\left(\ell_{1}\right)$ cannot be larger than the total amount of non-network coded and network coded traffic a node $o\left(\ell_{1}\right)$ receives over the inverse link $\overline{\ell_{1}}$. To describe these $\mathrm{NC}$ constraints, we refer to the pair of links $\left(\ell_{3}, \ell_{2}\right)$ and NC variable $y_{f_{1}, f_{2}}\left(\ell_{3}, \ell_{2}\right)$ in Fig. 2. In this network, the amount of network coded traffic $y_{f_{1}, f_{2}}\left(\ell_{3}, \ell_{2}\right)$ cannot be larger than the amount of non-network coded traffic the node $b$ receives over the inverse link $\ell_{4}$ for flow $f_{2}$. In general, the total amount of network coded traffic transmitted over the link $\ell_{3}$ by node $b$ cannot be larger than the total amount of non-network coded and network coded traffic node $b$ receives over the inverse link $\bar{\ell}_{3}$. The same must hold for the link $\ell_{2}$ in Fig. 2. The RHS of (14) shows the total amount of all flows that is received by a node $o\left(\ell_{1}\right)$ and restricts the total amount of encoded flows that can be transmitted over a link $\ell_{1}$.

The additional network coding constraints (15) on the variables $y_{f_{1}, f_{2}}\left(\ell_{1}, \ell_{2}\right)$ state that network coding between flows $f_{1}$ and $f_{2}$ for a node $n=o\left(\ell_{1}\right)=o\left(\ell_{2}\right)$ is not allowed if a node $n$ is the source or destination node for any flow $f_{1} \in \mathcal{F}$.

By solving this problem for a given network and a given transmit power, we obtain not only the value of the objective function but also the optimal schedule $\boldsymbol{\alpha}$ and the optimal vectors $\boldsymbol{x}, \boldsymbol{y}$. The JRS-NC problem in (10)-(17) when
$U\left(\left\{\lambda_{f}\right\}_{f \in \mathcal{F}}\right)=\min \left\{\lambda_{f}\right\}_{f \in \mathcal{F}}$ is a linear program, which is computationally hard to solve. Its complexity and size as compared to the JRS baseline problem is very high.

\section{Computational Technique}

Although, the JRS-NC problem is formulated as a LP, it is computationally hard to solve it optimally. The maximum number of links $|\mathcal{L}|$ in a network with $N$ nodes is of the order of $O\left(N^{2}\right)$. In the JRS problem the number of variables is $O\left(|\mathcal{L}||\mathcal{F}|+|\mathcal{L}|^{M^{\prime}}\right)$, where $M^{\prime}$ is the size of the largest ISet. The size of the JRS-NC problem is much larger than that of the JRS problem due to the added NC variables $y_{f_{1}, f_{2}}\left(\ell_{1}, \ell_{2}\right)$ and the larger cardinality of $\mathcal{I}$. The number of variables is $O\left(|\mathcal{L}||\mathcal{F}|+|\mathcal{L}|^{2}|\mathcal{F}|^{2}+|\mathcal{L}|^{M^{\prime \prime}}\right)$, where $M^{\prime \prime}$ is the maximum ISet size in the JRS-NC problem and typically $M^{\prime \prime}>M^{\prime}$.

The difficulty in solving this type of problem is the large number of variables and ISets. However, in an optimal solution, not all ISets are needed, as the number of non-zero (basis variables) $\alpha_{s}$ is at most $|L|+1$ [14]. Hence, only a small number of ISets or columns are needed. Knowing this fact, a technique similar to that presented in [14] is developed to reduce the number of ISets required to obtain exact results. This computational technique is based on the column generation method.

In this method, at each iteration, a reduced cost is computed for each off-basis column (or ISet) and only the columns that have the best potential to improve the objective function, based on this cost, are added to the Linear Program. The reduced costs for an ISet $s$ is given by:

$$
\begin{aligned}
r_{s} & =\zeta+\sum_{\ell_{1} \in \mathcal{L}_{1}(s)} v_{\ell_{1}}^{\prime}+\sum_{\left(\ell_{1}, \ell_{2}\right) \in \mathcal{L}_{2}(s)} v_{\ell_{1}, \ell_{2}}^{\prime \prime} \\
\mathcal{L}_{1}(s) & =\left\{\ell_{1} \in s / \nexists \ell_{2} \in s, o\left(\ell_{1}\right)=o\left(\ell_{2}\right), \ell_{1} \neq \ell_{2}\right\} \\
\mathcal{L}_{2}(s) & =\left\{\left(\ell_{1}, \ell_{2}\right) \in s^{2} / o\left(\ell_{1}\right)=o\left(\ell_{2}\right), \ell_{1} \neq \ell_{2}\right\},
\end{aligned}
$$

where $\zeta, v^{\prime}$ and $v^{\prime \prime}$ are the dual variables for (16), (12) and (13) respectively. Instead of adding a single off-basis column (ISet) with the most negative cost per iteration, at each iteration multiple off-basis columns that satisfy $r_{s}<\xi$, where $\xi<0$ is some specified tolerance, are added into the Linear Program. After addition of new off-basis columns, the JRS-NC problem is solved using the simplex method and if strictly negative reduced costs for off-basis columns are found, an additional iteration of the modified column generation is performed. However, if no strictly negative reduced costs are found, the current solution is optimal and the algorithm terminates.

\section{NUMERICAL RESULTS AND ENGINEERING INSIGHTS}

In this section, we obtain exact numerical results for Wireless Mesh Networks (WMN). Typically, in a mesh network each node has a flow destined to a gateway node $\mathcal{G}$ (i.e., an uplink flow) and $\mathcal{G}$ has a flow destined to each node (i.e., a downlink flow). In practice, a downlink flow has a larger rate than an uplink flow. To take this remark in consideration, we study a weighted optimization problem for $\max -\min$ throughput since it was shown in [7] that it is a reasonable objective for a managed WMN. Specifically, in the JRS-NC problem (resp. JRS problem), we replace (10) (resp. (3)) by 
(21) and add new constraint (22), where $w_{f}$ is a per flow weighting factor.

$$
\begin{gathered}
\max _{\boldsymbol{\alpha}, \boldsymbol{x}, \boldsymbol{y}} \lambda \\
0 \leq \lambda \leq \frac{\lambda_{f}}{w_{f}}, \quad \forall f \in \mathcal{F}
\end{gathered}
$$

In the following, we consider two types of weighting factors: equal weighting $1: 1$ and unequal weighting $1: 2$, with $w_{f}=2$ for all downlink flows to ensure that downlink flows obtain twice the throughput of uplink flows. By solving the JRS-NC problem, we obtain an optimal network configuration for routing and scheduling such that $\lambda$ is maximized that results in total throughput per node $\Lambda=2 \lambda$ for $1: 1$ and $\Lambda=3 \lambda$ for 1:2. All our results are in terms of $\Lambda$.

The optimal solutions for the JRS-NC problem with NC allowed between any two flows and at any node are denoted by full $N C$ and the results for the baseline JRS problem without any NC are denoted by JRS.

We also study variants of the JRS-NC problem which are denoted as: $G W-N C$, Bidirectional $N C$ and Bidirectional $G W-N C$ respectively. In the first variant $(G W-N C)$, only the nodes that are adjacent to the gateway can perform network coding, i.e., the variables $y_{f_{1}, f_{2}}\left(\ell_{1}, \ell_{2}\right)$ are only defined if $d\left(\ell_{1}\right)=\mathcal{G}$ or $d\left(\ell_{2}\right)=\mathcal{G}$ (the number of nodes that are adjacent to the gateway is a function of the transmit power $P)$. In the second variant (Bidirectional NC), network coding is permitted only between bidirectional flows, where two flows $f_{1}$ and $f_{2}$ are defined as bidirectional if $o\left(f_{1}\right)=d\left(f_{2}\right)$ and $o\left(f_{2}\right)=d\left(f_{1}\right)$. In this third variant Bidirectional $G W$ $N C$, only the nodes adjacent to the gateway can perform network coding, and even then, only between bidirectional flows. These variants are of interest for two reasons. First, they help isolate the interplay between the network topology and network coding that provides most of the gain. Second, they result in formulations that involve considerably fewer variables, and thus significantly reduce the complexity of the optimization and computation time.

We provide solutions for medium size networks with a total of $N=20$ nodes, where $N-1$ nodes are randomly and uniformly placed in a rectangle and a gateway node is deterministically placed for type-A scenario in a corner and for type-B in the middle of a grid. A scenario with a gateway in a corner is typical of sectored systems with directional antennas, while a gateway in the middle is typical on nonsectored systems.

We obtained results for 10 network realizations for each type-A and type-B scenario. We consider the following channel gain model for simplicity only: $G_{o(\ell), d(\ell)}=\left(d / d_{0}\right)^{-\mu}$, where $d_{0}$ is the reference distance, $\mu$ is the path loss exponent and $d$ is the distance between the transmitter and the receiver. The physical layer parameters for all network realizations are as follows: $N_{0}=-100 \mathrm{dBm}, d_{0}=0.1, \mu=3, \beta=6.4 \mathrm{~dB}$. In the following, $P_{S H}$ denotes the minimum power required for all nodes to communicate with the gateway node in single hop and $\bar{P}_{S H}$ denotes the minimum single hop power averaged over 10 realizations.

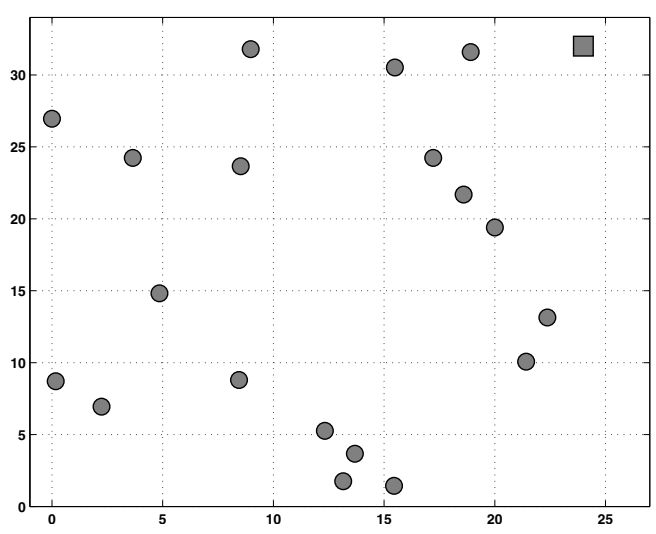

Fig. 3. A type-A network realization

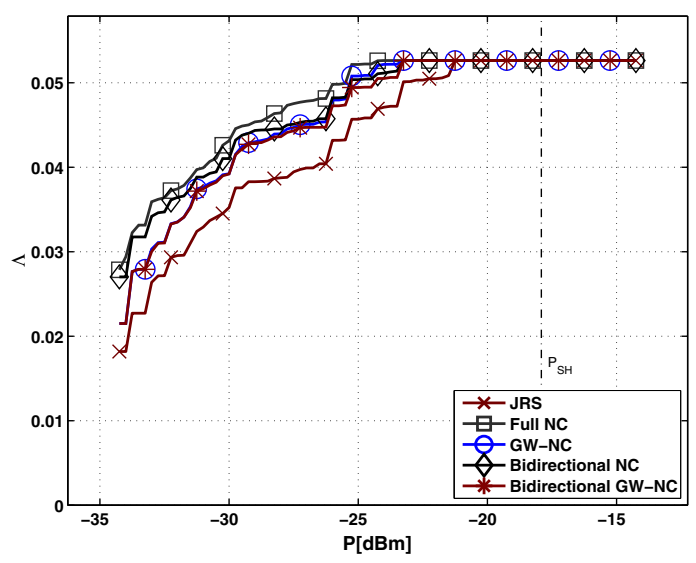

Fig. 4. $\max -\min$ total throughput per node vs transmit power $P$, Uplink+Downlink (1:1) for the network in Fig. 3

For the network realization shown in Fig. 3, we show the throughput $\Lambda$ as a function of the transmit power $P$ for the case 1:1 in Fig. 4 and for 1:2 in Fig. 5, respectively. Fig. 6 and Fig.7 show the relative gains in throughput averaged over the 10 type-A network realizations for 1:1 and 1:2 cases, respectively. These results show the relative gains of using the full NC and the three NC variants with respect to the JRS solutions (without network coding). We also provide results for one realization of a type-B network in Fig. 8 and in Fig. 9 for another realization for the 1:1 case and show the relative gains in throughput averaged over the 10 type-B networks realizations in Fig.10 and Fig.11 for the 1:1 and 1:2 cases, respectively. These results show that:

- Simple XOR-based network coding (e.g. Full NC) can yield interesting gains at low to medium transmit power (as high as $35 \%$ when the gateway is in the corner and as high as $15 \%$ when the gateway is in the middle). Note that these are average gains over 10 network realizations, and the actual gain of a particular network can differ significantly from the average.

- Full network coding enables us to reach the maximum achievable per node throughput of $1 / N$ (where $N$ is the number of nodes not counting the gateway) at significantly lower transmit power (on average about $3 \mathrm{~dB}$ lower) 


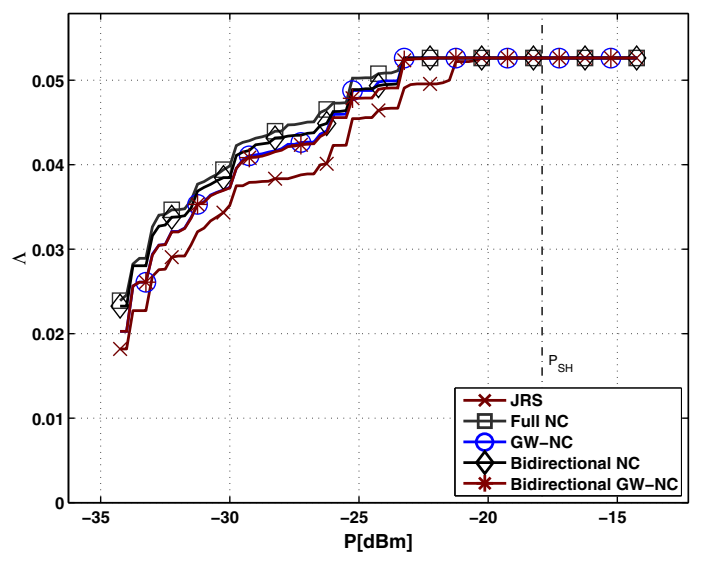

Fig. 5. $\max -\min$ total throughput per node vs transmit power $P$, Uplink+Downlink (1:2), for the network in Fig. 3

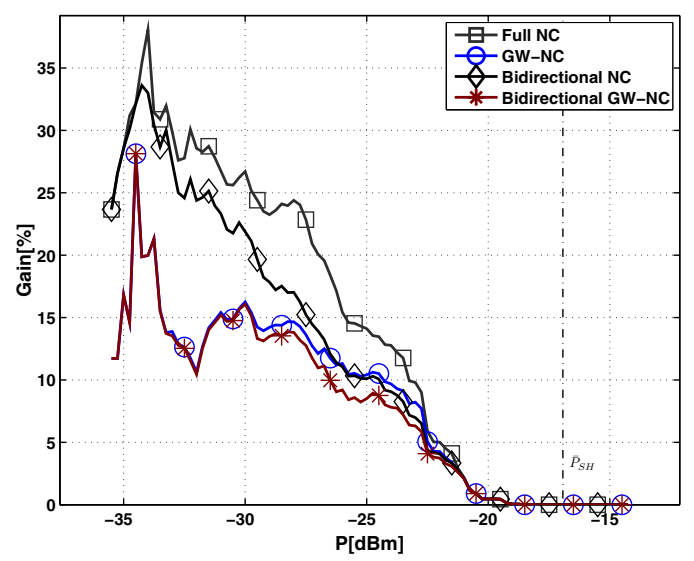

Fig. 6. Average relative gain in throughput vs transmit power $P$, Uplink+Downlink (1:1), for the 10 type-A realizations

when the gateway is at the corner (this remark is not valid for the cases where the gateway is in the middle). Note that, as discussed in [7], [14], using multihop communications enables a mesh network operator to offer this maximum achievable throughput at a transmit power $P$ which is much lower than $P_{S H}$. What we show here is that with simple XOR-based network coding we can even decrease further the power $P$ at which an operator can offer this throughput for the case when the gateway is at the corner.

- Network coding is less effective at high power since the number of hops required for optimum routing decreases and hence there are less opportunities for NC. Network coding is also less effective if the gateway is placed close to the nodes (type-B scenario), since it reduces the number of potential intermediate nodes for NC. The number of potential intermediate nodes between the gateway and the nodes in a random network is topology dependent. Therefore, it is possible to have almost no NC coding gains in such network realizations as shown in Fig.9. The relative gains are even smaller for unequal uplink to downlink flow rates in type-B networks due to

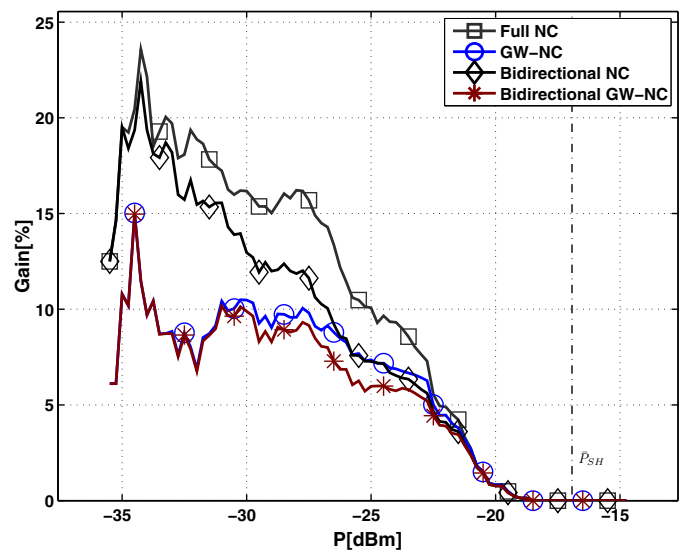

Fig. 7. Average relative gain in throughput vs transmit power $P$, Uplink+Downlink (1:2), for the 10 type-A realizations

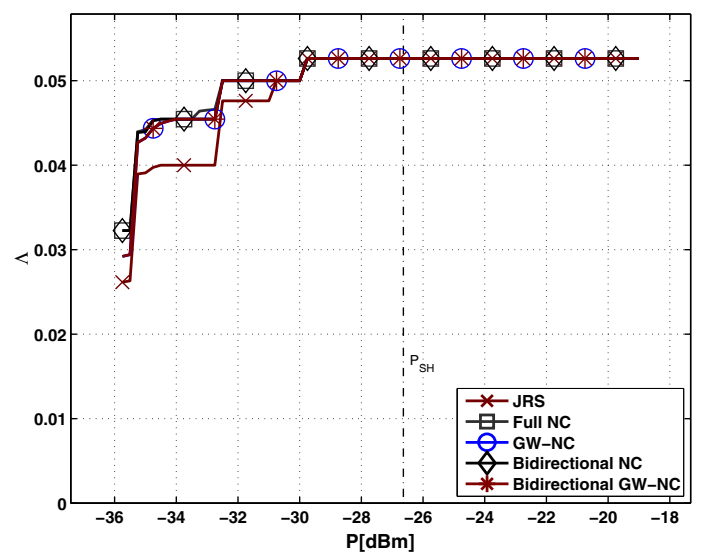

Fig. 8. $\max -\min$ total throughput per node vs transmit power $P$, Uplink+Downlink (1:1), for one of the type-B network realizations

reduced $\mathrm{NC}$ opportunities between flows as can be seen by comparing Fig. 11 and Fig 10.

- Restricting $\mathrm{NC}$ only at nodes adjacent to the gateway yields a significant portion of the gain over the performance without NC. We attribute this gain to the fact that nodes near the gateway are the bottleneck for network performance. Thus increasing the efficiency of flows at nodes that are distant from the gateway provides little benefit, while doing so at nodes near the gateway results in a direct improvement in performance.

- Limiting network coding to bidirectional flows does not perform identically to the Full NC case.

- When there is an imbalance in the flows as in the 1:2 case, network coding does not perform as well since there are less opportunities for XORing opposing flows due to the constraint in (14) and the unequal amount of incoming traffic at relay nodes.

\section{CONCLUSION}

In this paper, we jointly configure a wireless multihop network in terms of routing and conflict-free scheduling with network coding for unicast flows. We formulate a comprehen- 


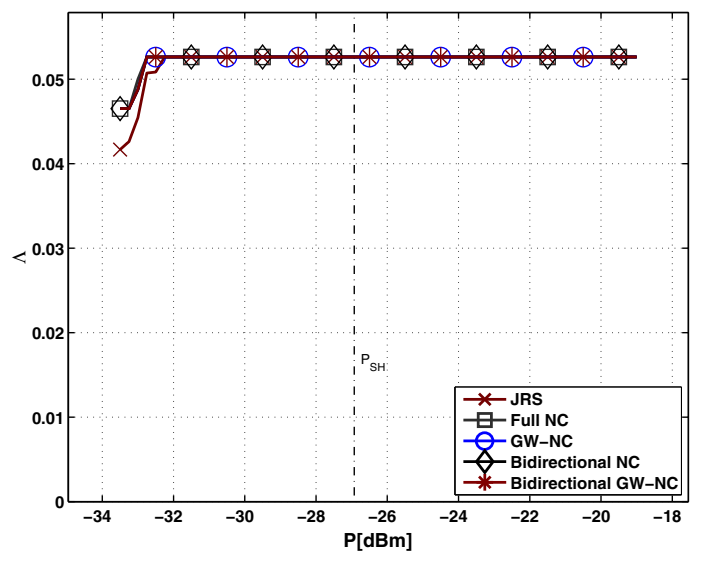

Fig. 9. $\max -\min$ total throughput per node vs transmit power $P$, Uplink+Downlink (1:1), for another of the type-B network realizations

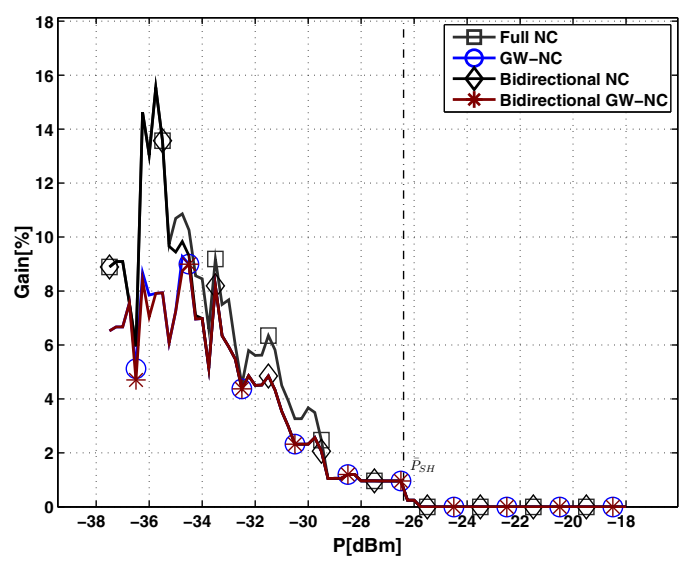

Fig. 10. Average relative gain in throughput vs transmit power $P$, Uplink+Downlink (1:1), for the 10 type-B realizations

sive mathematical optimization problem for the joint routing, scheduling, and network coding under a realistic physical interference model. This formulation is general and can be used for any given network to study the achievable throughput given a utility function. We provide exact solutions for medium size networks and quantify the throughput gains that can be achieved by using the XOR-based network coding without opportunistic listening. The results that we obtain are interesting since jointly optimizing routing, scheduling, and network coding can in some cases yield significant throughput gains at low to medium transmit power while in other cases it might yield little gains. At high transmission powers, as single hop transmission to the gateway is optimal, no relaying is needed, and hence network coding provides no advantage.

\section{REFERENCES}

[1] R. Ahlswede, N. Cai, S. R. Li, and R. W. Yeung, "Network information flow," IEEE Trans. on Information Theory, vol. 46, 2000.

[2] A. Capone, G. Carello, I. Filippini, S. Gualandi, and F. Malucelli, "Routing, scheduling and channel assignment in wireless mesh networks: optimization models and algorithms," Ad Hoc Netw. 2010, vol. 8.

[3] J. Groenkvist and A. Hansson, "Comparison between graph-based and interference-based STDMA scheduling," in International Symposium on Mobile Ad Hoc Networking and Computing, 2001, pp. 255-258.

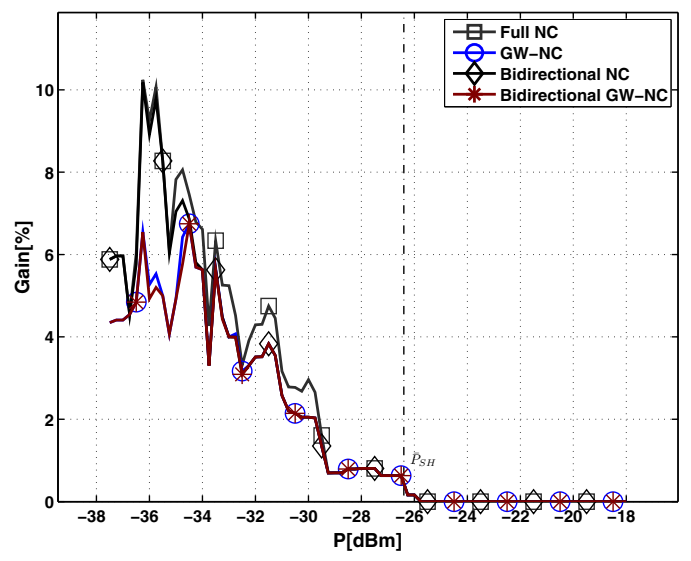

Fig. 11. Average relative gain in throughput vs transmit power $P$, Uplink+Downlink (1:2), for the 10 type-B realizations

[4] P. Gupta and P. Kumar, "The capacity of wireless networks," IEEE Transactions on Information Theory, vol. 34, pp. 910-917, 2000.

[5] A. Iyer, C. Rosenberg, and A. Karnik, "What is the right model for wireless channel interference?" IEEE Transactions on Wireless Communications, vol. 8, no. 5, pp. 2662 - 2671, 2009.

[6] K. Jain, J. Padhye, V. N. Padmanabhan, and L. Qiu, "Impact of interference on multi-hop wireless network performance,' in MobiCom 2003.

[7] A. Karnik, A. Iyer, and C. Rosenberg, "Throughput-optimal configuration of fixed wireless networks," IEEE/ACM Trans. in Networking, vol. 16, no. 5, pp. 1161-1174, 2008.

[8] S. Katti, D. Katabi, W. Hu, H. Rahul, and M. Medard, "The importance of being opportunistic: practical network coding for wireless environments," invited paper, Allerton Annual Conference on Communication, Control and Computing, 2005.

[9] S. Katti, H. Rahul, W. Hu, D. Katabi, M. Médard, and J. Crowcroft, "XORs in the air: practical wireless network coding," SIGCOMM Comput. Commun. Rev., vol. 36, no. 4, pp. 243-254, 2006.

[10] A. Khreishah, C.-C. Wang, and N. Shroff, "Rate control with pairwise intersession network coding," IEEE/ACM Trans. on Networking,, vol. 18, no. 3, pp. $816-829,2010$.

[11] K. Li and X. Wang, "Cross-layer design of wireless mesh networks with network coding," IEEE Trans. on Mobile Computing, vol. 7, 2008.

[12] S.-Y. Li, R. Yeung, and N. Cai, "Linear network coding," IEEE Transactions on Information Theory, vol. 49, no. 2, pp. $371-381$, feb. 2003.

[13] J. Liu, D. Goeckel, and D. Towsley, "Bounds on the throughput gain of network coding in unicast and multicast wireless networks," IEEE J.Sel. A. Commun., vol. 27, no. 5, pp. 582-592, 2009.

[14] J. Luo, C. Rosenberg, and A. Girard, "Engineering wireless mesh networks: joint scheduling, routing, power control and rate adaptation," IEEE/ACM Trans. in Networking, vol. 8, 2010.

[15] R. Nelson and L. Kleinrock, "Spatial TDMA: A collision-free multihop channel access protocol, impact of interference on multi-hop wireless," IEEE Trans. on Commununications, vol. 33, no. 9, pp. 934-944, 1985.

[16] Y. E. Sagduyu and A. Ephremides, "Cross-layer optimization of MAC and network coding in wireless queueing tandem networks," IEEE Transactions on Information Theory, vol. 54, no. 2, pp. 554-571, 2008.

[17] S. Sengupta, S. Rayanchu, and S. Banerjee, "An analysis of wireless network coding for unicast sessions: the case for coding-aware routing," in INFOCOM, 2007.

[18] C.-C. Wang and N. Shroff, "Pairwise intersession network coding on directed networks," IEEE Trans. on Information Theory, vol. 56, 2010.

[19] J. Zhang, H. Wu, Q. Zhang, and B. Li, "Joint routing and scheduling in multi-radio multi-channel multi-hop wireless networks," in INFOCOM'05.

[20] J. Zhang and Q. Zhang, "Cooperative network coding-aware routing for multi-rate wireless networks," in INFOCOM, 2009. 